\title{
Predictors of Social Mobilization Speed
}

\author{
Jeff Alstott \\ Stuart Madnick \\ Chander Velu
}

Working Paper CISL\# 2013-04

March 2013

Composite Information Systems Laboratory (CISL)

Sloan School of Management, Room E62-422

Massachusetts Institute of Technology

Cambridge, MA 02142 


\title{
Predictors of Social Mobilization Speed
}

\author{
Jeff Alstott ${ }^{1,2}$, Stuart Madnick $^{3}$, Chander Velu ${ }^{4}$
}

${ }^{1}$ Section on Critical Brain Dynamics, National Institute of Mental Health, Bethesda, Maryland, USA

${ }^{2}$ Behavioral and Clinical Neuroscience Institute, Departments of Experimental Psychology and Psychiatry, University of Cambridge, Cambridge, UK

${ }^{3}$ Sloan School of Management and School of Engineering, Massachusetts Institute of Technology, Cambridge, MA, USA

${ }^{4}$ Cambridge Judge Business School, University of Cambridge, Cambridge, UK

\section{Running Header: $\quad$ Predictors of Social Mobilization Speed}

Keywords: $\quad$ social mobilization, mobilization speed, demographics

Acknowledgement: The authors would like to thank Anton Phillips for operational and financial support as the general manager at Langley Castle, Sunny Cheung for designing and implementing the web site software, and Wei Pan for insights and suggestions based on his experience with the Red Balloon experiment. The authors would also like to thank Roy Welsch and Frank Harrell for their help in statistical interpretation. Contact Stuart Madnick for access to the contest data.

\footnotetext{
Abstract

Mobilization across social networks is becoming increasingly influential, but little is known about what traits of individuals and their relationships affect their speed of mobilization between them. We ran a global social mobilization contest and recorded personal traits of the participants and those they mobilized. We examined how those traits influenced the speed of mobilization. Individuals mobilized faster when they heard about the contest directly from the contest organization, and decreased in speed when hearing from less personal source types (e.g. family vs. media). Mobilization was faster when the mobilizer and the mobilized heard about the contest through the same source type, and slower when both individuals were in different countries. Females mobilized other females faster than males mobilized other males. Mobilization was faster with young recruiters and old recruits, and slower with old recruiters and young recruits. These findings suggest ways to optimize the speed of social mobilization.
} 


\section{INTRODUCTION}

Social mobilization is a large-scale movement to engage people's participation in achieving a specific goal through self-reliant efforts, and has broad impact in human society (1). Examples include social movements to change policies in governments $(2,3)$ and spontaneously self-organizing teams to run search and rescue operations $(4,5)$. As organizations and firms are increasingly turning to large-scale crowdsourcing to solve a variety of problems (6), creating social mobilization tasks is a way for them to direct many people towards a goal. While recent research has identified ways to engineer tasks to mobilize a large number of people (1), here we show factors that influence the speed of mobilization.

Social mobilization typically spreads on an existing social network. The spatial or topological path of activity spreading has been well-described in such contexts as search and information diffusion (7-10). However, the speed of activity spread can also be influential, particularly in time-sensitive domains like rescue operations or campaigning prior to elections. This rate of spreading is heterogeneous and its distribution is heavy-tailed for various types of human communication $(11,12)$. The demographic factors influencing speed have also been well-characterized for such diffusion-like processes as the spread of product adoption and musical tastes (13-15). In the case of social mobilization, however, in which individuals are actively recruiting others for a purpose, our understanding of the predictors of speed of mobilization are still at a nascent stage.

Here we show how personal traits of the individuals in social networks influence the speed of mobilization. We consider the traits of both the mobilizing and mobilized individuals separately as well as the relationships between both individuals' sets of traits jointly. In this way we consider the effect of the nodes of the social network (the traits of the individuals) as well as those of the links (the interactions of the individuals' traits). A better understanding of the determinants of social mobilization speed may enable engineering of mobilization scenarios in order to achieve a particular objective. For example, individuals could be organized more quickly towards a task or purpose by targeting the right elements of the social network.

\section{RESULTS}

We ran a global contest involving time-critical social mobilization in 2011, inspired by the Red Balloon Challenge organized by the Defense Advanced Research Projects Agency (DARPA) in the United States in 2009 (1). The contest was for Langley Castle Hotel in Northumberland, United Kingdom. The task was to find five knights in parks throughout the United Kingdom on a particular weekend, each with an ID code written on their shield. Contest participants had financial incentive to form large teams by recruiting new members, who then recruited other members, and so on (example team structure, Fig. 1). The first registered participant to correctly report the position of a knight was awarded $£ 1,000$. The person who recruited that participant received $£ 500$, and their recruiter received $£ 250$, and so on. Any team that as a whole found more than one knight would be awarded a $£ 250$ bonus, given to the team founder to distribute as desired. Additionally, the team leaders of the first, second, and third largest teams received $£ 1,000$, 500 , and $£ 250$ respectively. Two of these knights were "cyber knights", present not in the physical parks themselves but in Google Maps or Google Earth. This allowed for participants outside of the UK to readily participate, 
and indeed over $30 \%$ of participants with geographic information were from outside the UK.

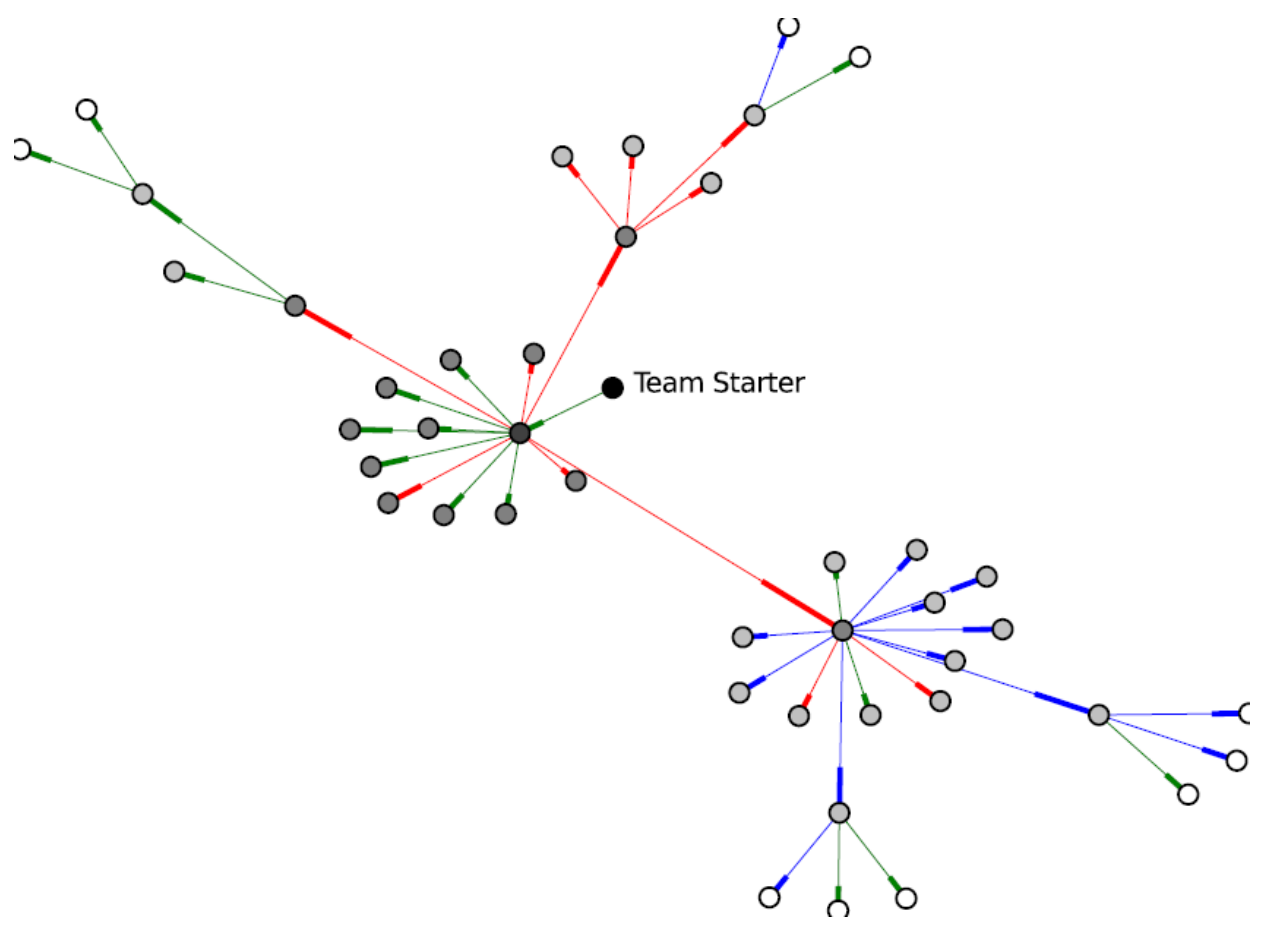

Figure 1. An example team growing from "parents" to "children", with different parent-child mobilizations having different types of links. The team starter's icon is black, and the future members decrease in shade as their generation in the team increases. Blue links indicate the parent and child heard about the contest through the same type of source (ex. friends). Red links indicate the parent and child heard through different types of sources (ex. family vs. the media). Green links indicate one or both participants did not give information on this trait. This example team was the $4^{\text {th }}$ largest in the contest.

\section{Mobilization Speed}

A total of 1,089 participants registered, and of these, 941 participants ("children") were recruited by an existing team member (their "parent"). We examined these parent-child recruitments' speed and factors influencing them. When participants registered on the Langley Castle Hotel website to join a team they provided personal information about themselves and how they heard about the contest. We also recorded when participants registered on the website. The difference in time between when one participant registered and when a child they recruited registered was the speed of mobilization across that social connection, and is similar to the inter-signup time metric used in Pickard et al. (1). We modeled this speed of mobilization with a Cox proportional hazard model, which has been used extensively to describe spreading of epidemics and subsequently adopted to study diffusion processes on social networks, such as product adoption (13). This model included the effects of the pairings of the parent's and child's ages, their genders, the sources from which they heard about the contest, their geographic locations, and the number of children they had in the contest. A hazard function is the likelihood of an event occurring after some time $t$. In our hazard model, the hazard function at time $t$ is the likelihood of a child registering for the contest $t$ units of time after their parent has registered. The influence of a particular factor, such as geographic location, is observed by how much higher or lower the 
hazard is in the presence of that factor relative to a baseline. This increase or decrease in hazard to baseline is expressed as a hazard ratio. Higher hazard ratios reflect higher likelihoods of registering for the contest at all times $t$, which indicate a faster social mobilization speed. Lower hazard ratios, conversely, indicate slower social mobilization speed, through lower likelihoods of registering for all times $t$.

The traits and relationships examined were the sources from which participants heard about the contest, participants' demographics (age, gender, and location), the number of children the participants recruited, and the timing of individuals' registration relative to both others in their team and the contest timeline. The role of these traits in social mobilization has not been previously studied

The source from which the participant first heard about the contest influenced mobilization speed. Mobilization speed increased when the participant heard about the contest direct from Langley Castle (Fig. 2A). From the other categories of information source, the next highest speeds were from family members, then friends, down to the participant's organization or simply the media (difference of hazard ratios between "Langley Castle" and "Media", $\mathrm{p}<.01$; all statistical tests on hazard ratio differences are derived from $\chi^{2}$ tests). This trend suggests that a direct relationship between the participants and the organizers of the mobilization yields the fastest mobilization speeds. In the absence of a direct connection, more intimate or psychologically close sources of information produce faster social mobilization.
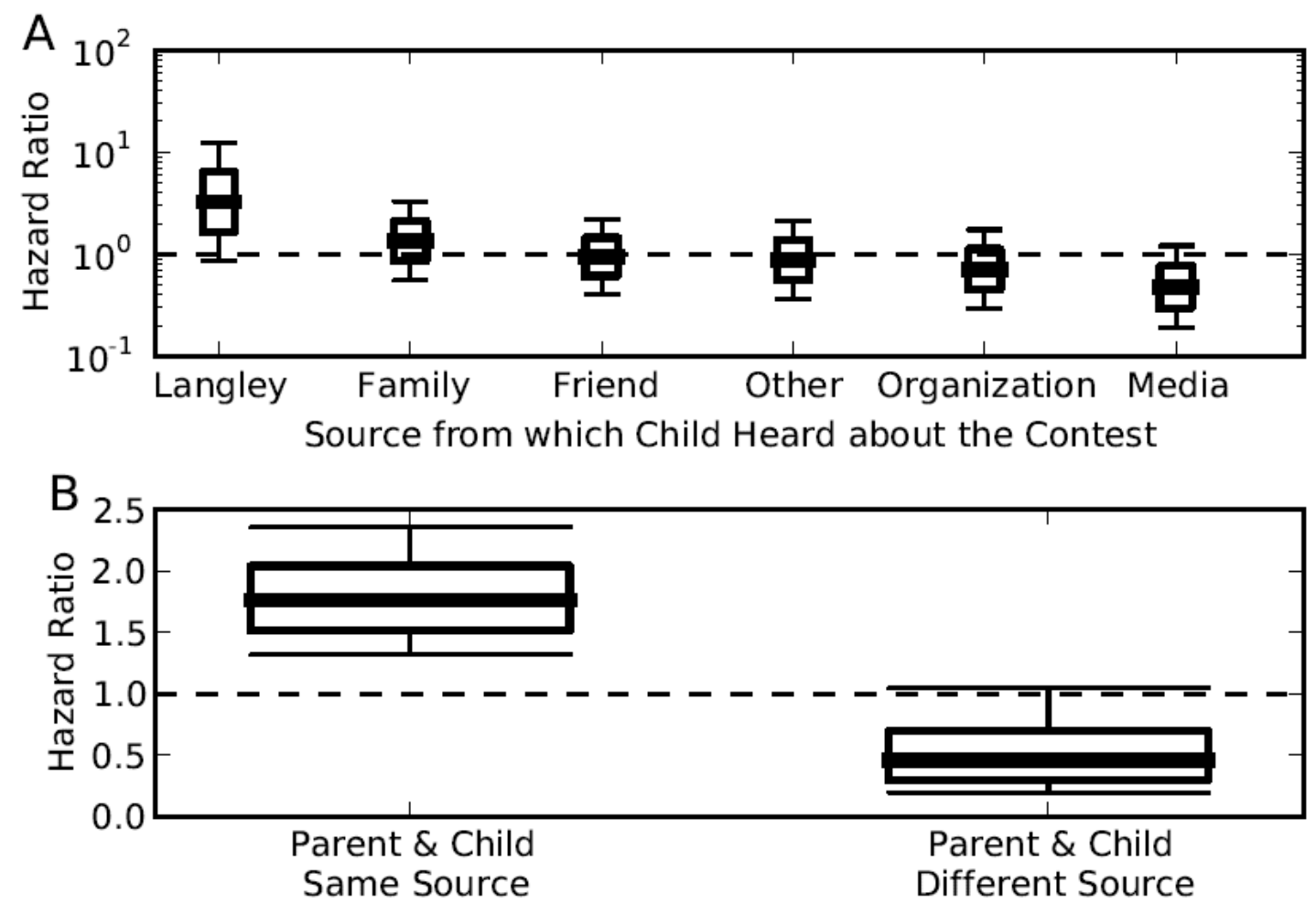

Figure 2. Where a participant heard about the contest affects mobilization speed. In all figures hazard ratios are the increase $(>1)$ or decrease $(<1)$ in likelihood of registering for the contest on a given day, reflecting an increase or decrease in mobilization speed. Boxes represent standard errors, and whiskers represent $95 \%$ confidence intervals. Unless otherwise noted, the reference rate (hazard 
ratio $=1$ ) is for participants who did not give data on that variable, or parent-child pairs in which at least one of the participants did not give data. (A) Mobilization speed is fastest when participants hear about the contest from Langley Castle directly, and decreases as the source is more psychologically distant from the participant. (B) Mobilization is faster when the parent and child heard about the contest through the same category of source than when they heard through different sources.

Mobilization speed increased when the child and parent heard about the contest through the same type of source (Fig. 2B).

Social mobilization speed was also lower when the parent and child were in different countries and fastest when they were in the same city (Fig. 3; p<.01). Our findings show that even in an era of increased telecommunications and "flattening" of the world, geographic proximity still holds considerable influence on how quickly teams mobilize.

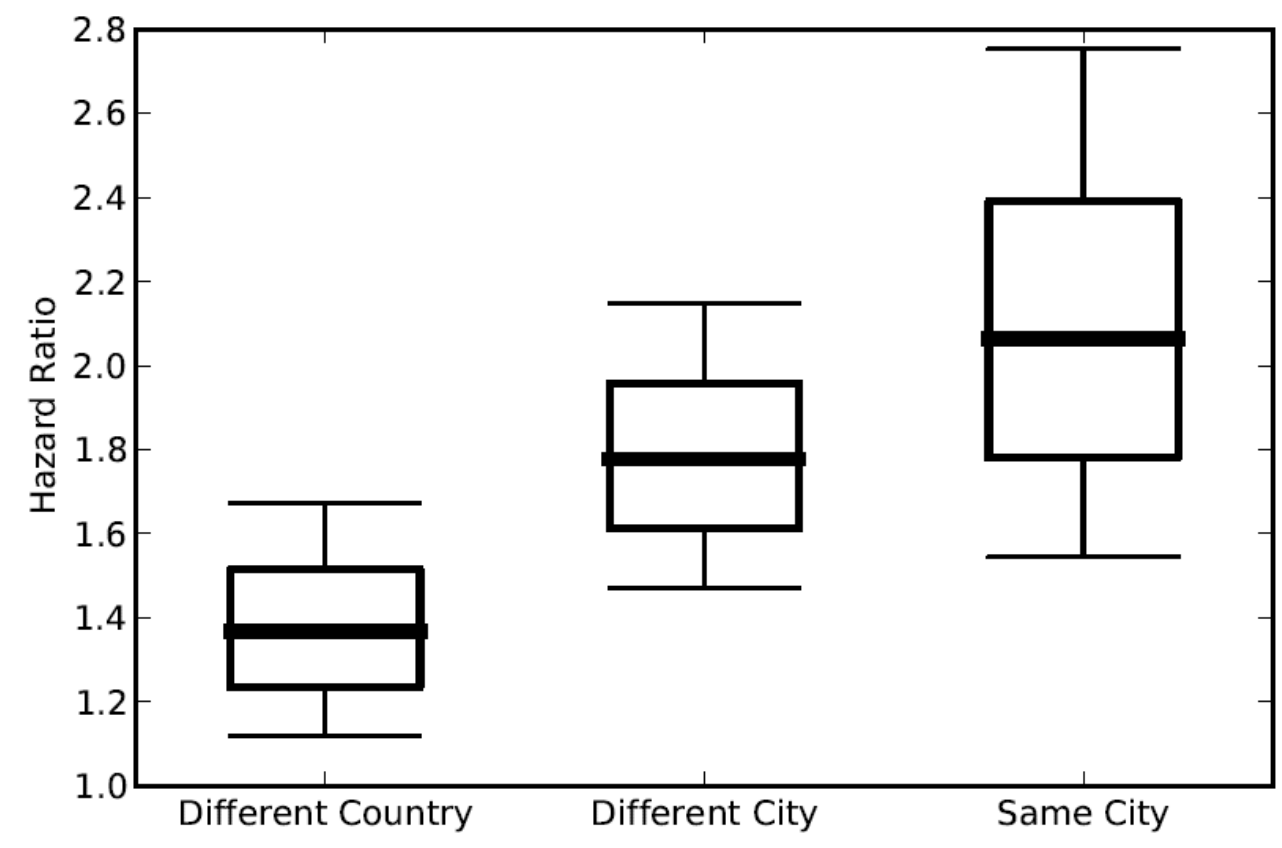

Figure 3. Geographically closer relationships have faster mobilization speed. Social mobilization is faster when the parent and child are in the same city, and slowest when they are in different countries.

Recent research on the role of gender in the speed of product adoption spread has yielded conflicting findings on whether males or females have greater influence or susceptibility to influence $(13,14)$. In the present social mobilization task, females mobilized each other faster than males mobilized each other (Fig. $4 ; \mathrm{p}<.05$ ). Females mobilizing males and males mobilizing females did not yield statistically significant different speeds $(\mathrm{p}>.05)$. 


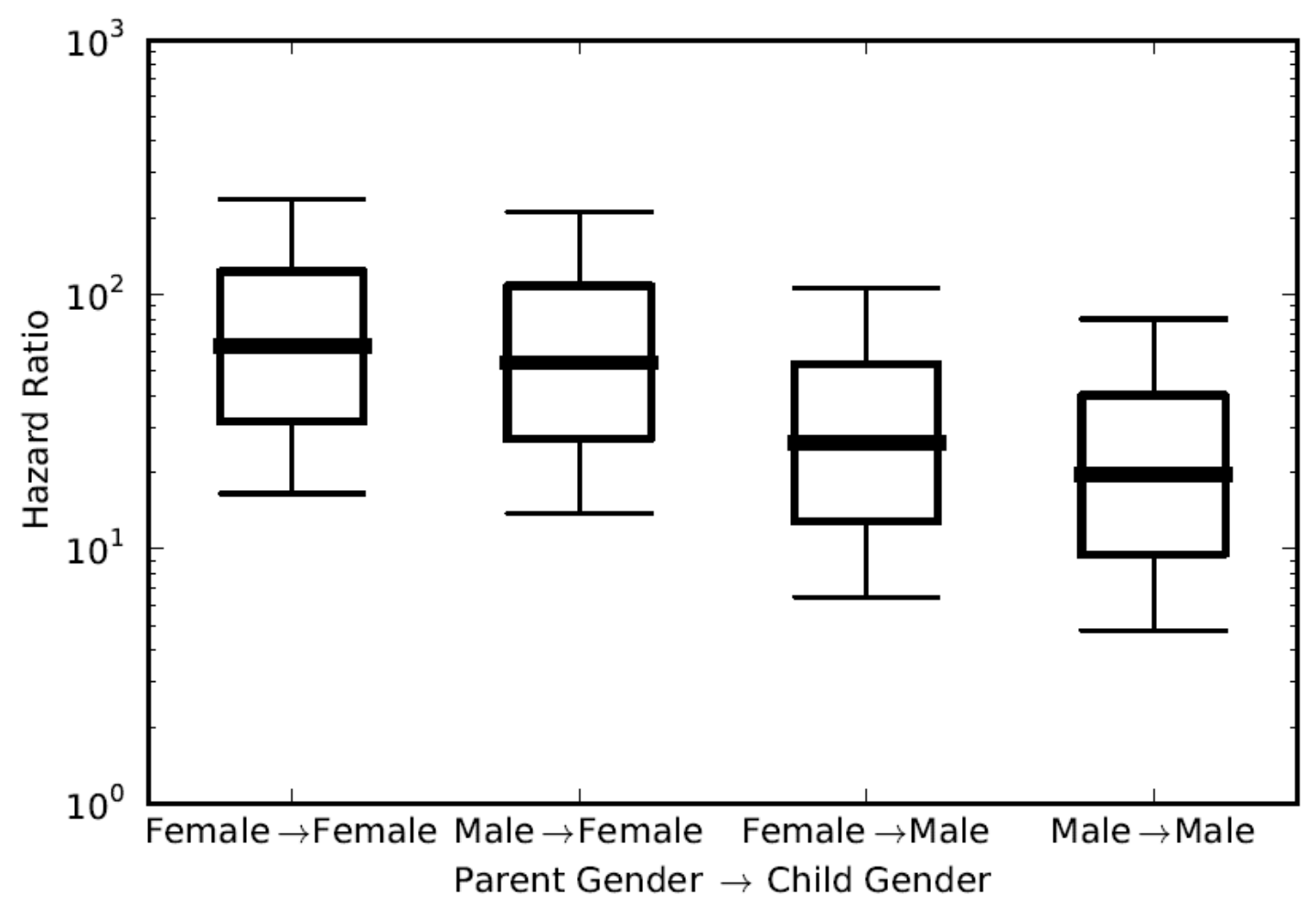

Figure 4. Females mobilize other females faster than males mobilize other males.

The effect of the parent's and child's ages on mobilization speed were pronounced. Participants' ages were binned into 20 -year ranges. Older children mobilized faster, regardless of the parent's age group (Fig. 5A). This finding is in contrast to the main effect of the child's age group, considered without an interaction with parent age, which shows younger children mobilizing faster (Fig. 5B). Similarly, older parents resulted in slower mobilization for any given child age group (Fig. 5C). This finding is again in contrast to the main effects of parent age, considered without any interaction with child age, which shows older parents mobilizing faster (Fig. 5D). These main effects mirror findings about age group's influence and susceptibility in product adoption (13). However, we show that in social mobilization the role of age is reversed when interaction effects are included. In particular, young parents and old children display fast mobilization, while old parents and young children display slow mobilization. 

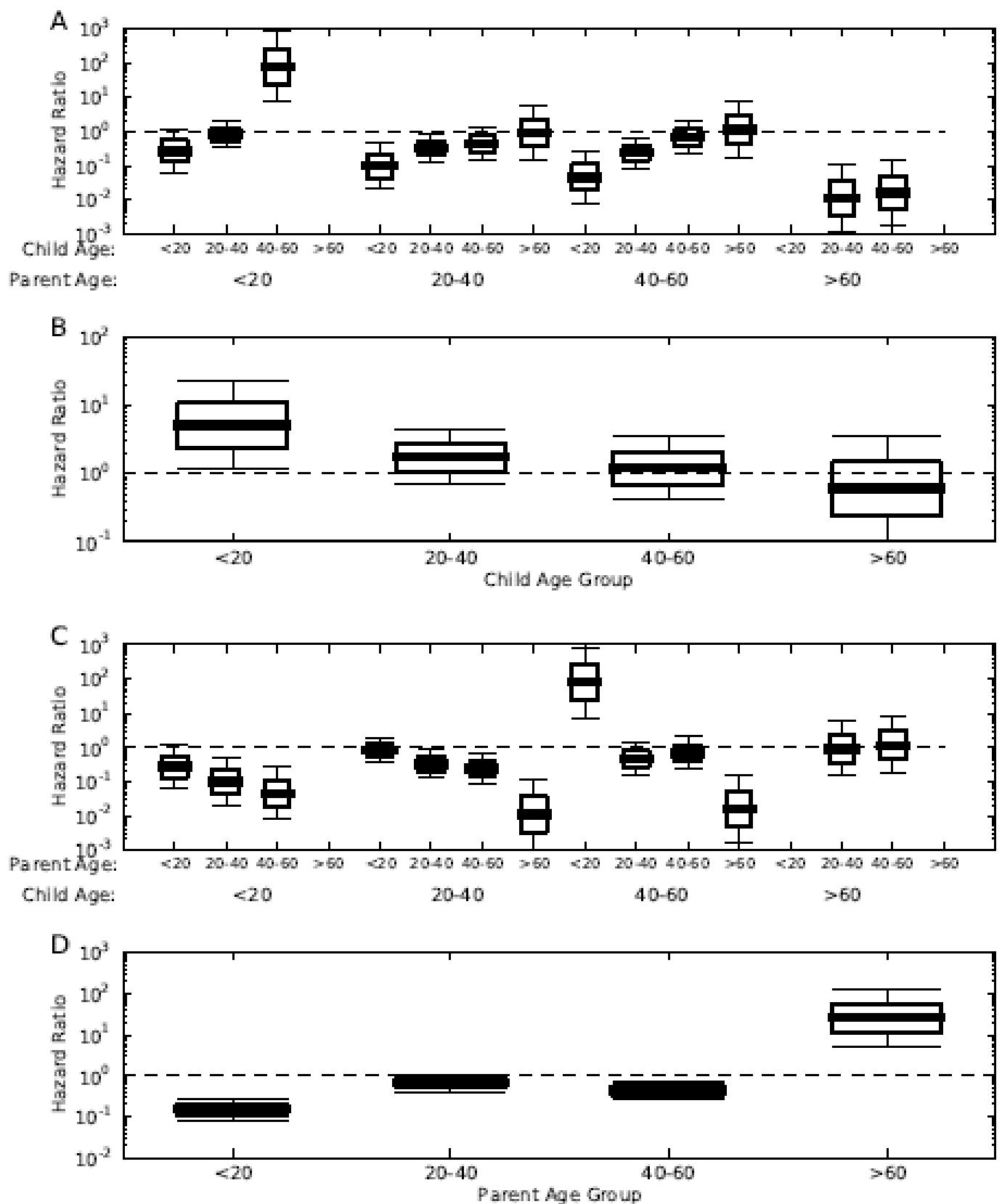

Figure 5. Older children and younger parents have faster mobilization speeds. (A) The interaction of parent and child age group on mobilization time, grouped by the parent's age. For any given parent age group, mobilization speed increases with the child's age. (B) The main effect of the child's age group on mobilization speed. (C) The interaction of parent and child age group on mobilization time, grouped by the child age. For any given child age group, mobilization speed decreases with the parent's age. (D) The main effect of the parent's age group on mobilization speed.

On average, as a parent had more children, the slower each of those children mobilized (Fig. 6A). In contrast, the more future children a participant would have, the faster that participant mobilized. While causality obviously does not allow a participant's number of future children to directly affect his or her own mobilization speed, the statistical relationship indicates that the people that mobilize quickly also recruit more children, independent of other factors. 

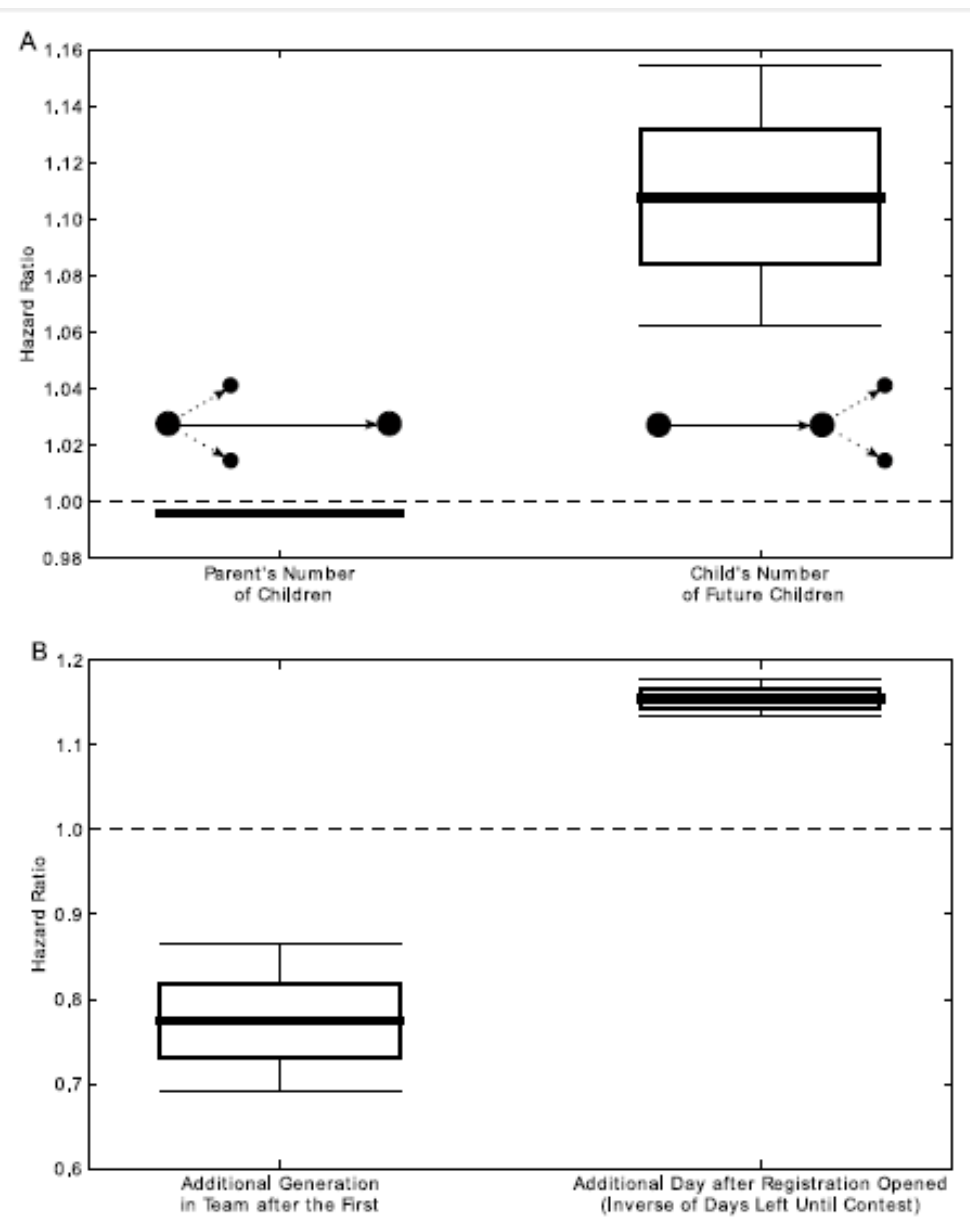

Figure 6. Additional children, additional generations, and time left in the contest all affect mobilization speed. (A) Parents with more children mobilize others slightly slower, while those who themselves mobilize quickly will have more children. Each additional child the parent has beyond one (hazard ratio $=1$ ) slightly slows down the mobilization speed of his or her children (see diagram inset left). Conversely, the child's mobilization speed is increased for each additional future child he or she will have beyond zero (see diagram inset right). (B) As a team grows with generations of parents recruiting children, each additional generation beyond the first (hazard ratio $=1$ ) slows down mobilization speed. In contrast, the further in time the recruiting happens (i.e. closer to the contest date), the faster the mobilization speeds.

As teams grew, parents mobilized children, who then in turn became parents mobilizing their own children. This process created "generations" of mobilization within a team. Each additional generation had slower mobilization relative to the one before it (Fig. 6B). In contrast, every additional day after registration opened (meaning one less day until the contest began) the social mobilization speed increased, on average.

\section{Team Dynamics}

Of the 1,089 participants, 148 started their own team. Of the teams, 97 did not mobilize any other team members, leaving 51 teams that recruited new participants. In these teams, 152 participants were parents, mobilizing at least one other participant. These parents mobilized 941 children. The mean team size was 7.36, and the mean size of teams larger than 1 was 19.45. The distributions of both team size and the number of children per parent had heavy tails (Fig. 7). 

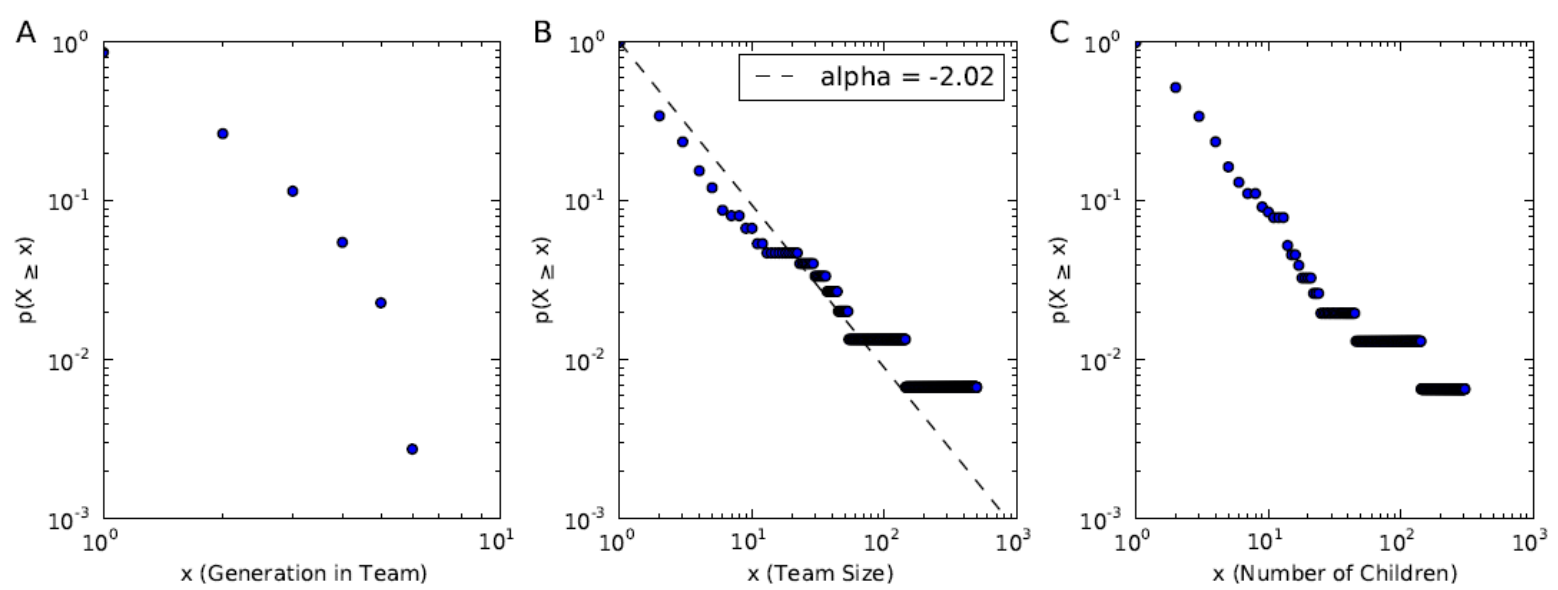

Figure 7. Several team dynamics have modest agreement with power law distributions. Using the same social mobilization incentive system used in the present study, previous research suggested the distributions of the number of generations in a team, team size, and a parent's number of children all followed power laws, with $\alpha$ of $3.00,1.96$ and 1.69, respectively (1). We used the statistical methods of (16) to find only weak to modest support for discrete power laws on these metrics, though with similar values for $\alpha$. All distribution plots are complementary cumulative distributions (survival functions). (A) Generation in the team of the 941 children (generation $0=$ Team starters). The power law distribution fit was not significantly better than the exponential (LLR: -.22, p>.1) or lognormal distributions (LLR: -.36, p>.1). (B) Team size. There were 148 teams, with 51 recruiting additional members beyond the founder. The power law fit was preferred over an exponential (LLR: 58.53, $\mathrm{p}<.01$ ), but was no better of a fit than a lognormal (LLR:.01, p>.9) (C) Number of children for each participant. There were 1,089 participants, with 152 mobilizing at least one child. The power law fit was better than that of an exponential (LLR: 61.45, $\mathrm{p}<.02$ ), but was not a stronger fit than the lognormal distribution (LLR:-.04, p>.9)

Previous research on a contest using the same incentive system as the present study had suggested the team dynamics followed power laws, extremely heavy tailed probability distributions (1). Such power laws are notable because they imply the existence of extremely large events, such as a social mobilization that grows to encompass the entire global social network. We examined the team dynamics in the present study using rigorous statistical methods (16), described in Methods, and found only weak to modest support for power laws (Fig. 7).

\section{DISCUSSION}

As social mobilization becomes increasingly influential, the ability to engineer and influence the dynamics of mobilization will become ever more important within society. The present findings provide a novel and nuanced understanding of the predictors of the speed of mobilization on social networks. These insights may allow for speed-optimization of social mobilization tasks, such as political campaigning, marketing, and others. The speed of recruitment can be increased by focusing on a number of simple elements of a mobilization task. For example, targeting all-female groups instead of all-male groups would lead to faster mobilization in a disease prevention campaign trying to quickly propagate best practices against a new virus. Encouraging specifically the young to recruit others, particularly those older than themselves, would accelerate the take-up in a new political campaign trying to rapidly build a base. Operating through family networks instead of work networks or mass media would speed up recruitment for a search and rescue operation. Encouraging participants around the globe to recruit largely within their own cities would hasten buy-in to 
a movement to build up a donations network in the wake of a natural disaster.

Such large-scale social mobilizations are becoming increasingly common and impactful, and often the speed of recruitment is critical to their success. For those organizing such mobilization tasks, a greater understanding of the factors driving mobilization speed can improve the odds of success. The predictors of social mobilization speed described here compose an initial set of relevant elements, and open the door for identification of additional factors. 


\section{Appendix: Data Collected and Methods of Analysis}

The contest was advertised by Langley Castle, through its web site langleycastle.com, newsletters, Facebook pages, email lists, and press releases. A copy of the master press release can be found at (17) and an amusing video is at (18). Participants registered on the contest website, where they were directed to give demographic information about themselves and how they heard about the contest. Participants could register with their email and making a password on the site, or alternatively through Facebook Connect. Registration on the website opened June 1, 2011. The competition started on July 2, 2011 at 9am and ended at $9 \mathrm{pm}$ on July 3, 2011. The "real" knights were in their assigned parks from roughly 9am to 9pm each day. The "cyber" knights were present on Google Maps and Google Earth all day.

Participants who registered using Facebook Connect could, at the end of the registration process, invite their Facebook friends to join the contest. Registered participants were also provided with a URL they could share with others to register through, which would automatically put those new participants on their parent's team. In addition to the URL, the participants were also given a number that other participants could enter manually to register as their child.

Participants submitted information on knights they found through a form on the website, which required the inclusion of a code unique to each knight printed on their shield. Knights that were already found were announced on the contest website.

\section{Personal information collected}

The participant's geographic location was inferred from their IP address. Participants whose IP addresses could not be localized to the city level were treated as not having geographic data. The participant registration form's personal information questions and response categories were:

"Heard about us from": Friend, Family member, Your organization, Langley Castle, Media, Other

"Gender": Male, Female

"Age range": < 20 years old, 20-40 years old, 40-60 years old, > 60 years old

Participants were not required to respond on any question. The number of children with available data on a category and the number of parent-child pairings in which both participants had available data on a category were:

"Heard about us from": 505, 426

"Gender": 774, 756

"Age range": 529, 475

Geographic information: 704, 637

\section{Proportional Hazards Model of Mobilization Speeds}

We modeled the speed of mobilization using a single failure, continuous time Cox proportional hazard model. The time modeled was the interval between when a parent and 
child registered, with the time interval beginning when the parent registered and "failure" when the child registered. The modeled hazard function is the likelihood of a child registering on a given day after the parent has registered. This is expressed relative to a baseline hazard function, creating a hazard ratio. Higher hazard ratios reflect higher likelihoods to register at a given time, which when multiplied successively across time speed up the point at which "failure" (registration) will occur. Thus, high hazard ratios indicate fast mobilization speed. Conversely, low hazard ratios extend the time until registration will occur, reflecting slower mobilization speed.

The factors included in the model were:

- the parent's and child's ages and their interaction

- the parent's and child's genders and their interaction

- the type of source from which the child heard about the contest

- if the parent and child heard about the contest from the same type of source

- if the parent and child were in different countries, different cities in the same country, or the same city

- the number of children the parent had

- the number of children the child would have

- the generation the child was in the team

- the time since registration opened (the inverse of the time remaining until the contest began), which was expressed as the date the parent registered

With the child's traits described in a set $X_{*}$, and the parent's traits described in a set $X_{\mathrm{x}}$, and the child's and parent's ages and genders represented in the set $S\left(X_{c}, X_{p}\right)$, our model had the form:

$$
\lambda_{u}\left(t, X_{:}, X_{\tau}\right)=\lambda_{u}(t) \exp \left[X_{*} \beta^{c}+X_{\mathrm{v}} \beta^{p}+S\left(X_{c}, X_{p}\right) \beta^{*, p}\right]
$$

With $\dot{\lambda}_{\mathrm{J}}$ as the baseline hazard function as a function of time since the parent's registration $t$, and $\lambda_{*}\left(t_{1}, X_{:}, X_{\tau}\right)$ as the hazard for a child at time $t$ with traits $X_{z}$ and whose parent had traits $X_{\mathrm{x}}$. The coefficient $\beta^{\mathrm{c}}$ is the effect of the child's traits on the hazard, $\beta^{p}$ is the effect of the parent's traits, and $\beta^{c, p}$ the effect of the interaction of their age and gender traits.

\section{Power Law Tests of Team Dynamics}

We used the statistical methods of (16) to evaluate whether several features of team dynamics were well-described by power law distributions. These features were the distributions of the number of generations in a team, team size, and a parent's number of children. The statistical methods included using a loglikelihood ratio (LLR) test between a best-fit power law (found through maximum likelihood methods) and an alternative distribution. A positive LLR indicates the power law fit is more likely, and a negative shows 
the alternative distribution is more likely. The significance of that LLR, however, is given by a p-value. A statistically insignificant LLR means the data does not clearly fit either of the candidate distributions more than the other. Lastly, the best-fit power law may not cover the entire distribution, but only be a good fit beyond a certain value, the $\mathrm{x}_{\min }$. The shape of these distributions does not impact the use of the Cox proportional hazards model for describing mobilization speed. 


\section{References}

1. Pickard G et al. (2011) Time-critical social mobilization. Science (New York, NY) 334:509-12.

2. Deutsch KW (1961) Social Mobilization and Political Development. The American Political Science Review 55:493-514.

3. Bond RM et al. (2012) A 61-million-person experiment in social influence and political mobilization. Nature 489:295-8.

4. Krakow G (2005) Ham radio operators to the rescue after Katrina. NBC News.

5. Hellerstein JM, Tennenhouse DL (2011) Searching for Jim Gray : A Technical overview. Communications of the ACM 54:77-87.

6. Afuah A, Tucci CL (2012) Crowdsourcing as a Solution to Distant Search. Academy of Management Review 37:355-375.

7. Watts DJ, Dodds PS, Newman MEJ (2002) Identity and Search in Social Networks. Science (New York, NY) 296:1302-5.

8. Onnela J-P et al. (2007) Structure and tie strengths in mobile communication networks. Proceedings of the National Academy of Sciences of the United States of America 104:7332-6.

9. Pajevic S, Plenz D (2012) The organization of strong links in complex networks. Nature Physics 8:429-436.

10. Kleinberg JM (2000) Navigation in a small world. Nature 406:845.

11. Iribarren JL, Moro E (2009) Impact of Human Activity Patterns on the Dynamics of Information Diffusion. Physical Review Letters 103.

12. Malmgrem RD, Stouffer DB, Motter AE, Amaral LAN (2008) A Poissonian explanation for heavy tails in e-mail communication. Proceedings of the National Academy of Sciences 105:18153-18158.

13. Aral S, Walker D (2012) Identifying Influential and Susceptible Members of Social Networks. Science 337:337-41.

14. Katona Z, Zubcsek PP, Sarvary M (2011) Network Effects and Personal Influences : The Diffusion of an Online Social Network. Journal of Marketing Research 48:425443.

15. Lewis K, Gonzalez M, Kaufman J (2012) Social selection and peer influence in an online social network. Proceedings of the National Academy of Sciences of the United States of America 109:68-72.

16. Clauset A, Shalizi CR, Newman MEJ (2009) Power-law distributions in empirical data. SIAM Review 51.

17. Langley Knights Press Release Available at: http://web.mit.edu/smadnick/www/Langley/LangleyKnightPressRelease-master.pdf

18. Langley Knights Newsreel Available at: http://www.youtube.com/watch?v=B2Z7NULcurk 